PROCEEDINGS OF THE

AMERICAN MATHEMATICAL SOCIETY

Volume 135, Number 12, December 2007, Pages 3805-3813

S 0002-9939(07)08938-1

Article electronically published on September 7, 2007

\title{
THE KAC JORDAN SUPERALGEBRA: AUTOMORPHISMS AND MAXIMAL SUBALGEBRAS
}

\author{
ALBERTO ELDUQUE, JESÚS LALIENA, AND SARA SACRISTÁN
}

(Communicated by Martin Lorenz)

\begin{abstract}
In this note the group of automorphisms of the Kac Jordan superalgebra is described and used to classify the maximal subalgebras.
\end{abstract}

\section{INTRODUCTION}

Finite dimensional simple Jordan superalgebras over an algebraically closed field of characteristic zero were classified by V. Kac in 1977 [9] (see also Kantor [10], where a missing case is added). Among these superalgebras we find the ten dimensional Kac Jordan superalgebra, $K_{10}$, which is exceptional (see [12] and [17]) and plays a significant role (see [13]).

Here we are interested in describing the group of automorphisms of $K_{10}$ and, as a consequence, in classifying the maximal subalgebras of the Kac superalgebra over an algebraically closed field of characteristic zero.

In two previous works (see [6, [7]), the authors have given a description of maximal subalgebras of finite dimensional central simple superalgebras which are either associative or associative with superinvolution and also a description of maximal subalgebras of finite dimensional special simple Jordan superalgebras over an algebraically closed field of characteristic zero. These papers are thus in the spirit of previous work by E. Dynkin ([3], [4]), M. Racine ([14, [15]) or A. Elduque ([5]) on maximal subalgebras of different classes of algebras.

Let $F$ be a field of characteristic not two. This assumption will be kept throughout the paper. Recall that a superalgebra is a $\mathbb{Z}_{2}$-graded algebra $A=A_{\overline{0}} \oplus A_{\overline{1}}$ $\left(A_{\alpha} A_{\beta} \subseteq A_{\alpha+\beta} \forall \alpha, \beta \in \mathbb{Z}_{2}\right)$. If $a \in A_{\alpha}$ we say that $a$ is a homogeneous element and we use the notation $\bar{a}=\alpha$. Elements belonging to $A_{\overline{0}}$ are called even elements and the ones in $A_{\overline{1}}$ odd elements. A superalgebra $A$ is said to be nontrivial if $A_{\overline{1}} \neq 0$, and it is called simple if it contains no proper nontrivial graded ideals and $A^{2} \neq 0$.

Received by the editors September 6, 2005 and, in revised form, September 26, 2006.

2000 Mathematics Subject Classification. Primary 17C70.

Key words and phrases. Kac Jordan superalgebra, automorphism, maximal subalgebra.

The first and second authors have been supported by the Spanish Ministerio de Educación y Ciencia and FEDER (MTM 2004-081159-C04-02). The first author also acknowledges support of the Diputación General de Aragón (Grupo de investigación de Álgebra).

(C)2007 American Mathematical Society Reverts to public domain 28 years from publication 
A Jordan algebra is an algebra satisfying the following identities:

$$
\begin{aligned}
x y & =y x, \\
\left(x^{2} y\right) x & =x^{2}(y x) .
\end{aligned}
$$

The last identity can be written as $\left(x^{2}, y, x\right)=0$, where $(x, y, z)$ denotes the associator $(x y) z-x(y z)$. Following the standard procedure, a superalgebra is a Jordan superalgebra if its Grassmann envelope is a Jordan algebra. In particular, a Jordan superalgebra satisfies the identities

$$
\begin{gathered}
x y=(-1)^{\bar{x} \bar{y}} y x, \\
\sum_{\substack{\text { cyclic } \\
x, y, t}}(-1)^{(\bar{x}+\bar{z}) \bar{t}}(x y, z, t)=0
\end{gathered}
$$

for homogeneous elements $x, y, z, t$. (If the characteristic is $\neq 2,3$, these two identities characterize Jordan superalgebras.)

The even part of a Jordan superalgebra is a Jordan algebra, while the odd part is a Jordan bimodule for the even part.

Associative superalgebras are just $\mathbb{Z}_{2}$-graded associative algebras, but note that Jordan superalgebras are not $\mathbb{Z}_{2}$-graded Jordan algebras.

The following examples of Jordan algebras will be useful in the sequel:

i) Let $A$ be an associative algebra over a ground field of characteristic $\neq 2$. The new operation $a \cdot b=\frac{1}{2}(a b+b a)$ defines a structure of a Jordan algebra on $A$. It is denoted by $A^{+}$.

ii) Let $V$ be a vector space over $F$ with a nondegenerate symmetric bilinear form $():, V \times V \rightarrow F$. The direct sum $F 1+V$ with the product $(\lambda 1+$ $v)(\mu 1+w)=(\lambda \mu+(v, w)) 1+(\lambda w+\mu v)$ is a Jordan algebra, and it is called the Jordan algebra of a nondegenerate bilinear form.

Simple finite dimensional Jordan algebras over an algebraically closed field were classified by P. Jordan, J. von Neumann, E. Wigner ([8]) and by A. Albert ([1]). The examples given above are two of the four types of algebras given in that classification.

Also the following examples of Jordan superalgebras will be needed later on:

1) $J=K_{3}$, the Kaplansky superalgebra:

$$
\begin{aligned}
& J_{\overline{0}}=F e, \quad J_{\overline{1}}=F x+F y, \\
& e^{2}=e, \quad e \cdot x=\frac{1}{2} x, \quad e \cdot y=\frac{1}{2} y, \quad x \cdot y=e .
\end{aligned}
$$

2) The family of superalgebras $J=D_{t}$, with $t \in F \backslash\{0\}$ :

$$
\begin{aligned}
& J_{\overline{0}}=F e+F f, \quad J_{\overline{1}}=F u+F v, \\
& e^{2}=e, \quad f^{2}=f, \quad e \cdot f=0, \quad e \cdot u=\frac{1}{2} u, \quad e \cdot v=\frac{1}{2} v, \quad f \cdot u=\frac{1}{2} u, \\
& f \cdot v=\frac{1}{2} v, \quad u \cdot v=e+t f .
\end{aligned}
$$

3) $J=K_{10}$, the Kac superalgebra, which will be described in more detail in the following section.

4) The superalgebra of a superform: Let $V=V_{\overline{0}} \oplus V_{\overline{1}}$ be a graded vector space over $F$, and let $($,$) be a nondegenerate supersymmetric bilinear$ superform on $V$, that is, a nondegenerate bilinear map that is symmetric on $V_{\overline{0}}$, skewsymmetric on $V_{\overline{1}}$, and $V_{\overline{0}}, V_{\overline{1}}$ are orthogonal relative to $($,$) .$ Now consider $J_{\overline{0}}=F e+V_{\overline{0}}, J_{\overline{1}}=V_{\overline{1}}$ with $e \cdot x=x, v \cdot w=(v, w) e$ for every $x \in J, v, w \in V$. This superalgebra, $J$, is called the superalgebra of a superform. 
5) $A^{+}$, with $A$ an associative superalgebra over a field of characteristic not 2 , where the product operation in $A$ has been changed to $a_{i} \cdot b_{j}=\frac{1}{2}\left(a_{i} b_{j}+\right.$ $\left.(-1)^{i j} b_{j} a_{i}\right)$.

As we have mentioned at the beginning of this Introduction, V. Kac and I. Kantor gave the classification of nontrivial simple Jordan superalgebras. The four examples given above are also examples of simple Jordan superalgebras, assuming in 3) that the characteristic is not 3 , and in 4 ) that $A$ is a simple associative superalgebra (see [18]).

In this paper, our purpose is to describe the group of automorphisms of $K_{10}$ and also to classify its maximal subalgebras.

\section{KAC SUPERALGEBRA}

The Kac superalgebra, $K_{10}=J_{\overline{0}} \oplus J_{\overline{1}}$, is a Jordan superalgebra with 6-dimensional even part and 4-dimensional odd part:

$$
J_{\overline{0}}=\left(F e+F a+F b+\sum_{i=1}^{2} F c_{i}\right) \oplus F f, \quad J_{\overline{1}}=\sum_{i=1}^{2}\left(F p_{i}+F q_{i}\right)
$$

and product given in Table 1 .

TABLE $1 . K_{10}$

\begin{tabular}{c|cccccc|cccc} 
& $e$ & $a$ & $b$ & $c_{1}$ & $c_{2}$ & $f$ & $p_{1}$ & $p_{2}$ & $q_{1}$ & $q_{2}$ \\
\hline$e$ & $e$ & $a$ & $b$ & $c_{1}$ & $c_{2}$ & 0 & $\frac{1}{2} p_{1}$ & $\frac{1}{2} p_{2}$ & $\frac{1}{2} q_{1}$ & $\frac{1}{2} q_{2}$ \\
$a$ & $a$ & $4 e$ & 0 & 0 & 0 & 0 & $p_{1}$ & $p_{2}$ & $-q_{1}$ & $-q_{2}$ \\
$b$ & $b$ & 0 & $-4 e$ & 0 & 0 & 0 & $q_{1}$ & $q_{2}$ & $-p_{1}$ & $-p_{2}$ \\
$c_{1}$ & $c_{1}$ & 0 & 0 & 0 & $2 e$ & 0 & 0 & $q_{1}$ & 0 & $p_{1}$ \\
$c_{2}$ & $c_{2}$ & 0 & 0 & $2 e$ & 0 & 0 & $q_{2}$ & 0 & $p_{2}$ & 0 \\
$f$ & 0 & 0 & 0 & 0 & 0 & $f$ & $\frac{1}{2} p_{1}$ & $\frac{1}{2} p_{2}$ & $\frac{1}{2} q_{1}$ & $\frac{1}{2} q_{2}$ \\
\hline$p_{1}$ & $\frac{1}{2} p_{1}$ & $p_{1}$ & $q_{1}$ & 0 & $q_{2}$ & $\frac{1}{2} p_{1}$ & 0 & $a+2(e-3 f)$ & $2 c_{1}$ & $b$ \\
$p_{2}$ & $\frac{1}{2} p_{2}$ & $p_{2}$ & $q_{2}$ & $q_{1}$ & 0 & $\frac{1}{2} p_{2}$ & $-a-2(e-3 f)$ & 0 & $-b$ & $-2 c_{2}$ \\
$q_{1}$ & $\frac{1}{2} q_{1}$ & $-q_{1}$ & $-p_{1}$ & 0 & $p_{2}$ & $\frac{1}{2} q_{1}$ & $-2 c_{1}$ & $b$ & 0 & $a-2(e-3 f)$ \\
$q_{2}$ & $\frac{1}{2} q_{2}$ & $-q_{2}$ & $-p_{2}$ & $p_{1}$ & 0 & $\frac{1}{2} q_{2}$ & $-b$ & $2 c_{2}-a+2(e-3 f)$ & 0
\end{tabular}

This basis of $K_{10}$ is obtained by using the one in [16, p. 378] with $a=v_{1}+v_{2}$, $b=v_{1}-v_{2}, c_{1}=v_{3}, c_{2}=v_{4}, p_{1}=x_{1}-y_{2}, p_{2}=x_{2}+y_{1}, q_{1}=x_{1}+y_{2}, q_{2}=x_{2}-y_{1}$.

From [11] we know that $J_{\overline{0}}=J(V, Q) \oplus F f$, where $J(V, Q)$ is the Jordan algebra of a nondegenerate bilinear form $Q, e$ is the identity element in $J(V, Q)$, and $V$ is the vector space with basis $\left\{a, b, c_{1}, c_{2}\right\}$. We will use this presentation in Section 4 .

In 22 G. Benkart and A. Elduque gave a realization of $K_{10}$ which enables us to check directly that it is indeed a Jordan superalgebra. We introduce this construction, which will be very useful for us in Section 3.

First we pick up the Kaplansky superalgebra, $K_{3}$, over the field $F$ and we define the following supersymmetric bilinear form (that is, the even and odd parts are 
orthogonal and the form is symmetric in the even part and alternating in the odd part):

$$
(e \mid e)=\frac{1}{2}, \quad(x \mid y)=1, \quad\left(\left(K_{3}\right)_{\overline{0}} \mid\left(K_{3}\right)_{\overline{1}}\right)=0 .
$$

Consider now the vector space over $F: F \cdot 1 \oplus\left(K_{3} \otimes_{F} K_{3}\right)$ and define on it the product:

$$
(a \otimes b)(c \otimes d)=(-1)^{\bar{b} \bar{c}}\left(a c \otimes b d-\frac{3}{4}(a \mid c)(b \mid d) 1\right)
$$

with $a, b, c, d \in K_{3}$ homogeneous elements, and where 1 is a formal identity element. Then,

Theorem 2.1 (Benkart, Elduque). $K_{10}$ is isomorphic to $F \cdot 1 \oplus\left(K_{3} \otimes K_{3}\right)$ by means of the linear map given by:

$$
\begin{aligned}
e & \mapsto \frac{3}{2} \cdot 1-2 e \otimes e, & f & \mapsto-\frac{1}{2} \cdot 1+2 e \otimes e, \\
a & \mapsto-4 x \otimes x-y \otimes y, & b & \mapsto-4 x \otimes x+y \otimes y, \\
c_{1} & \mapsto 2 x \otimes y, & c_{2} & \mapsto-2 y \otimes x, \\
p_{1} & \mapsto 4 x \otimes e-2 e \otimes y, & p_{2} & \mapsto-4 e \otimes x-2 y \otimes e, \\
q_{1} & \mapsto 4 x \otimes e+2 e \otimes y, & q_{2} & \mapsto-4 e \otimes x+2 y \otimes e .
\end{aligned}
$$

If we denote by $W$ the vector space $\left(K_{3}\right)_{\overline{1}}$, we notice that $V=W \otimes W$ is endowed with the bilinear nondegenerate symmetric form given by $b(s \otimes t, u \otimes v)=(s \mid u)(t \mid v)$. We note also that the corresponding decomposition of $\left(K_{10}\right)_{\overline{0}}$ into the direct sum of two simple ideals according to the one given above is $\left(K_{10}\right)_{\overline{0}}=I \oplus J$, where $I=F \cdot(-1 / 2+2 e \otimes e)$ and $J=F \cdot(3 / 2-2 e \otimes e) \oplus V$.

\section{Automorphisms of $K_{10}$}

Here by automorphism we mean automorphism of graded algebras.

It is easy to compute the group of automorphisms of the examples 1), 2) and 4) of Jordan superalgebras given in Section 1. So, with the notation of Section 2, $\operatorname{Aut}\left(K_{3}\right)=S p(W)$, the symplectic group (or also $\operatorname{Aut}\left(K_{3}\right)=S L(W)$, the special linear group). In addition, $\operatorname{Aut}\left(D_{t}\right)=S p(U)$, where $U$ is the vector space generated by $\{u, v\}$. If $J$ is the superalgebra of a superform, then $\operatorname{Aut}(J)=O(n, F) \oplus$ $S p(m, F)$, where $O(n, F)$ denotes the orthogonal group of $V_{\overline{0}}$, with $\operatorname{dim} V_{\overline{0}}=n$, related to $\left.()\right|_{,V_{\overline{0}} \times V_{\overline{0}}}$, and $S p(m, F)$ denotes the symplectic group of $V_{\overline{1}}$, with $\operatorname{dim} V_{\overline{1}}=m$, related to $\left.()\right|_{,V_{\overline{1}} \times V_{\overline{1}}}$.

In what follows in this section it will be assumed that $F$ is a field of characteristic $\neq 2,3$ such that $F^{2}=F$.

Let $C_{2}=\{1, \epsilon\}$ be the cyclic group of order $2\left(\epsilon^{2}=1\right)$ and consider the wreath product $G=S p(W) \curlywedge C_{2}$ (that is, $G$ is the semidirect product $(S p(W) \times S p(W)) \rtimes C_{2}$ and $(f, g) \epsilon=\epsilon(g, f)$ for any $f, g \in S p(W))$. 
The following maps are clearly group homomorphisms:

$$
\begin{aligned}
& \Psi: \operatorname{Aut}\left(K_{10}\right) \longrightarrow O(V, b) \\
& \varphi \quad \longmapsto \varphi \mid V \\
& \tilde{\Psi}: \quad G \quad \longrightarrow O(V, b) \\
& (f, g) \quad \longmapsto f \otimes g: W \otimes W \rightarrow W \otimes W \\
& s \otimes t \quad \mapsto f(s) \otimes g(t) \\
& \epsilon \quad \longmapsto \quad \hat{\epsilon} \quad: W \otimes W \rightarrow W \otimes W \\
& s \otimes t \quad-t \otimes s \\
& \Phi: \quad G \quad \longrightarrow \operatorname{Aut}\left(K_{10}\right) \\
& (f, g) \longmapsto \Phi_{(f, g)}:\left\{\begin{aligned}
1 & \rightarrow 1 \\
a \otimes b & \mapsto \tilde{f}(a) \otimes \tilde{g}(b)
\end{aligned}\right. \\
& \epsilon \quad \longmapsto \quad \delta: \quad\left\{\begin{aligned}
1 & \rightarrow 1 \\
a \otimes b & \mapsto(-1)^{\bar{a} \bar{b}} b \otimes a
\end{aligned}\right.
\end{aligned}
$$

where for any $f \in S p(W), \tilde{f}$ denotes the automorphism of $K_{3}$ such that $\tilde{f}(e)=e$, $\tilde{f}(w)=f(w) \forall w \in W$.

Note that $\Psi \circ \Phi=\tilde{\Psi}$.

Lemma 3.1. $\tilde{\Psi}$ is onto with $\operatorname{Ker} \tilde{\Psi}=\{ \pm(i d, i d)\}$.

Proof. Let $\{u, v\}$ be a symplectic basis of $W$ (so $(u \mid v)=1$ ). Then if $\alpha=u \otimes s+v \otimes t$ is an isotropic vector in $V$ (that is, $b(\alpha, \alpha)=0)$, then $0=b(\alpha, \alpha)=(s \mid t)$, so $s, t$ are linearly dependent. This shows that

$$
\text { \{isotropic vectors of } V\}=\{s \otimes t \mid s, t \in W\} .
$$

Now let us show that $\tilde{\Psi}$ is onto. For any $\varphi \in O(V, b), \varphi(u \otimes u)$ is isotropic $(u \otimes u$ is), so there are $s, t \in W$ with $\varphi(u \otimes u)=s \otimes t \neq 0$.

Take $f, g \in S p(W)$ with $f(s)=u, g(t)=u$. Hence $\varphi \in i m \tilde{\Psi}$ if and only if

$$
(f \otimes g) \circ \varphi=\tilde{\Psi}(f, g) \circ \varphi \in i m \tilde{\Psi},
$$

and, therefore, it can be assumed that $\varphi(u \otimes u)=u \otimes u$.

Now $\varphi(v \otimes v)=s \otimes t$ for some $s, t \in W$ with

$$
1=b(u \otimes u, v \otimes v)=b(\varphi(u \otimes u), \varphi(v \otimes v))=b(u \otimes u, s \otimes t)=(u \mid s)(u \mid t),
$$

and there exists $0 \neq \alpha \in F$ such that $(u \mid s)=\alpha,(u \mid t)=\alpha^{-1}$ or $\left(u \mid \alpha^{-1} s\right)=1$, $(u \mid \alpha t)=1$. Thus there exist $f, g \in S p(W)$ such that $f(u)=u, f(v)=\alpha^{-1} s$, $g(u)=u, g(v)=\alpha t$, and changing $\varphi$ to $\left(f^{-1} \otimes g^{-1}\right) \circ \varphi$ we may assume that $\varphi(u \otimes u)=u \otimes u, \varphi(v \otimes v)=v \otimes v$.

Then $\varphi(u \otimes v)$ is isotropic and orthogonal to both $u \otimes u$ and $v \otimes v$, so it is a scalar multiple of either $u \otimes v$ or $v \otimes u$. By using $\tilde{\Psi}(\epsilon)=\hat{\epsilon}$ we may assume that $\varphi(u \otimes v)=\gamma u \otimes v$ for some $0 \neq \gamma \in F$ and then, necessarily, $\varphi(v \otimes u)=\gamma^{-1} v \otimes u$. Let $\mu \in F=F^{2}$ with $\mu^{2}=\gamma$. Then $\varphi=f \otimes g$ where $f(u)=\mu u, f(v)=\mu^{-1} v$, $g(u)=\mu^{-1} u, g(v)=\mu v$, so that $\varphi \in i m \tilde{\Psi}$.

The assertion about the kernel is clear. 
Lemma 3.2. $\Psi$ is onto with $\operatorname{Ker} \Psi=\{i d, \tau\}$, where $\tau$ is the grading automorphism $\left(\tau(z)=(-1)^{\bar{z}} z\right)$ for any homogeneous $z \in K_{10}$.

Proof. Since $\Psi \circ \Phi=\tilde{\Psi}$ and $\tilde{\Psi}$ is onto, so is $\Psi$. Moreover, if $\varphi \in \operatorname{Aut}\left(K_{10}\right)$ belongs to the kernel of $\Psi$, then $\varphi \mid\left(K_{10}\right)_{\overline{0}}=I d$, so $\varphi\left(z_{\overline{0}} \cdot z_{\overline{1}}\right)=z_{\overline{0}} \cdot \varphi\left(z_{\overline{1}}\right)$ for any $z_{\overline{0}} \in\left(K_{10}\right)_{\overline{0}}$ and $z_{\overline{1}} \in\left(K_{10}\right)_{\overline{1}}$.

For any $s \in W, s \otimes e$ is, up to scalars, the unique odd element annihilated by $s \otimes W\left(\subseteq\left(K_{10}\right) \overline{0}\right)$; hence $\varphi(s \otimes e)=\beta s \otimes e$ for some $0 \neq \beta \in F$.

Take $t \in W$ with $(s \mid t)=1$. Then $\forall z \in W,(t \otimes z) \cdot(s \otimes e)=\frac{1}{2} e \otimes z$, so $\varphi(e \otimes z)=2(t \otimes z) \cdot \varphi(s \otimes e)=\beta(e \otimes z)$ for any $z \in W$. It follows that $\varphi \mid\left(K_{10}\right)_{\overline{1}}=$ $\varphi \mid(e \otimes W \oplus W \otimes e)=\beta I d$ and, since $\varphi$ is an automorphism, $\beta^{2}=1$, so $\varphi$ is either the identity or the grading automorphism $\tau$.

Theorem 3.3. $\Phi$ is an isomorphism.

Proof. Since $\Phi(-i d,-i d)=\tau, \Phi(\operatorname{Ker} \tilde{\Psi})=\operatorname{Ker} \Psi$. Now the assertion follows from the fact that $\Psi \circ \Phi=\tilde{\Psi}$, together with Lemmata 1 and 2 .

\section{Maximal subalgebras of $K_{10}$}

In what follows the word subalgebra will be used in the graded sense, so any subalgebra is graded. Also in this paragraph we consider that $K_{10}$ is a superalgebra over $F$, an algebraically closed field of characteristic $\neq 2,3$.

Theorem 4.1. Any maximal subalgebra of $K_{10}$ is, up to an automorphism of $K_{10}$, one of the following:

(i) $\left(K_{10}\right)_{\overline{0}}$.

(ii) The subalgebra with basis $\left\{e, f, a, p_{1}, p_{2}\right\}$.

(iii) The subalgebra with basis $\left\{e, f, a+b, c_{1}, p_{1}, q_{1}, p_{2}+q_{2}\right\}$.

(iv) The subalgebra with basis $\left\{e, f, a, b, c_{1}, p_{1}, q_{1}\right\}$.

Proof. Note that if $B$ is a maximal subalgebra of $K_{10}, 1=e+f \in B$, as $F 1+B$ is a subalgebra of $K_{10}$ and $B$ is an ideal in $F 1+B$. If $f \notin B$, then $B_{\overline{0}} \oplus F f=\pi_{I}\left(B_{\overline{0}}\right) \oplus$ $\pi_{J}\left(B_{\overline{0}}\right)$, which is a subalgebra of $\left(K_{10}\right)_{\overline{0}}$ (recall that $\left(K_{10}\right)_{\overline{0}}$ is the direct sum of the ideals $I$ and $J=F f$; here $\pi_{I}$ and $\pi_{J}$ denote the corresponding projections), and so the subalgebra generated by $B$ and $f$ equals $B \oplus F f$. Since $B$ is maximal, $B \oplus F f=K_{10}$, so, in particular, $B_{\overline{1}}=\left(K_{10}\right)_{\overline{1}}$, but $\left(K_{10}\right)_{\overline{1}}$ generates $K_{10}$, so $B=K_{10}$, a contradiction. Therefore $f \in B$, and hence $e=1-f \in B$ too.

We now use the description of $K_{10}$ given at the beginning of Section 2 and due to D. King. Then $B_{\overline{0}}=F e+F f+V_{0}$, with $V_{0}$ a vector subspace of $V$ and so, in order to study the maximal subalgebras of $K_{10}$, we analyze the possible dimensions of $V_{0}$.

(1) If $V_{0}=V$, then $B=\left(K_{10}\right)_{\overline{0}}$ is a maximal subalgebra of $K_{10}$.

(2) If $\operatorname{dim} V_{0}=1$, we have two possible situations: either the rank of $Q$ is 1 or it is 0 .

If $\operatorname{rank}\left(\left.Q\right|_{V_{0}}\right)=1$, using Witt's Theorem and Theorem 3.3 we can suppose that $V_{0}=F a$. Now $B_{\overline{1}}=P_{B}+Q_{B}$ with $P_{B}=\{p \in B: p a=p\}, Q_{B}=\{q \in B: q a=$ $-q\}$. If $\operatorname{dim} P_{B}=2$ we can check that $B=\left\langle e, f, a, p_{1}, p_{2}\right\rangle$ is a maximal subalgebra of $K_{10}$. Likewise if $\operatorname{dim} Q_{B}=2, B=\left\langle e, f, a, q_{1}, q_{2}\right\rangle$ is a maximal subalgebra of $K_{10}$, but there is an automorphism of $K_{10}$ applying $\left\langle e, f, a, p_{1}, p_{2}\right\rangle$ into $\left\langle e, f, a, q_{1}, q_{2}\right\rangle$ (the automorphism given by the isometry that applies $a$ to $-a$ and fixes $b, c_{1}, c_{2}$ ). If $\operatorname{dim} P_{B}=\operatorname{dim} Q_{B}=1$, then we can check that $\operatorname{dim} V_{0}>1$, a contradiction. If 
$P_{B}=0$, then $B \subseteq\left\langle e, f, a, q_{1}, q_{2}\right\rangle$, which is a maximal subalgebra, and we are in the case above.

If $\operatorname{rank}\left(\left.Q\right|_{V_{0}}\right)=0$, using Witt's Theorem and Theorem 3.3 we can suppose that $V_{0}=F c_{1}$. But $c_{1} B_{\overline{1}} \subseteq F p_{1}+F q_{1}$ and $B_{\overline{1}} B_{\overline{1}} \subseteq F c_{1}+F e+F f$, so $B_{\overline{1}} \subseteq\left\langle p_{1}, q_{1}\right\rangle$. It follows that $B=\left\langle e, f, c_{1}, p_{1}, q_{1}\right\rangle$ is not maximal since $B \varsubsetneqq\left\langle e, f, a, b, c_{1}, p_{1}, q_{1}\right\rangle$.

(3) If $\operatorname{dim} V_{0}=2$, then $\operatorname{rank}\left(\left.Q\right|_{V_{0}}\right)=0,1$ or 2 .

If $\operatorname{rank}\left(\left.Q\right|_{V_{0}}\right)=2$, we can suppose that $V_{0}=F a+F b$, again by Witt's Theorem and Theorem 3.3. As in (2), we have that $B_{\overline{1}}=P_{B}+Q_{B}$. If $\operatorname{dim} P_{B}=2$, then since $b P_{B}=Q_{B}$, it follows that $\operatorname{dim} Q_{B}=2$, and then $B=K_{10}$, a contradiction. If $\operatorname{dim} P_{B}=1$, then $\operatorname{dim} Q_{B}=1$, but since $B_{\overline{1}} B_{\overline{1}} \subseteq B_{\overline{0}}$, then either $B_{\overline{1}}=\left\langle p_{1}, q_{2}\right\rangle$ or $B_{\overline{1}}=\left\langle p_{2}, q_{1}\right\rangle$. This is a contradiction with $b B_{\overline{1}} \subseteq B_{\overline{1}}$. If $\operatorname{dim} P_{B}=0$, then $\operatorname{dim} Q_{B}=0$ and $B$ is not a maximal subalgebra because $B \subseteq\left(K_{10}\right)_{\overline{0}}$.

If $\operatorname{rank}\left(\left.Q\right|_{V_{0}}\right)=1$, then $V_{0}=F a+F c_{1}$ and again $B_{\overline{1}}=P_{B}+Q_{B}$. If $\operatorname{dim} P_{B}=2$, then $\operatorname{dim} Q_{B} \geq 1$ (because $c_{1} p_{1}=0$ and $c_{1} p_{2}=q_{1}$ ) and so $B=K_{10}$, a contradiction. If $\operatorname{dim} P_{B}=1$, then $\operatorname{dim} Q_{B}=1$ or 0 . If $\operatorname{dim} Q_{B}=1$, from $B_{\overline{1}} B_{\overline{1}} \subseteq B_{\overline{0}}$ we have that $Q_{B}=F q_{1}$ and for $0 \neq p \in P_{B}$ it follows that $p q_{1} \in V_{0}=F a+F c_{1}$, and therefore $p \in F p_{1}$. But in this case $B$ is not maximal since $B \varsubsetneqq\left\langle e, f, a, b, c_{1}, p_{1}, q_{1}\right\rangle$. Also, if $\operatorname{dim} Q_{B}=0$, then $P_{B}=F p_{1}$ and again $B$ is not maximal. Now if $\operatorname{dim} P_{B}=0$, then $\operatorname{dim} Q_{B}=1$, with $Q_{B}=\left\langle q_{1}\right\rangle$. But again $B$ is not maximal because $B \varsubsetneqq$ $\left\langle e, f, a, b, c_{1}, p_{1}, q_{1}\right\rangle$.

Finally, if $\operatorname{rank}\left(\left.Q\right|_{V_{0}}\right)=0$, we can suppose that $V_{0}=F \cdot(a+b)+F c_{1}$, by Witt's Theorem and Theorem 3.3. If $x=\alpha p_{1}+\beta p_{2}+\sigma q_{1}+\gamma q_{2} \in B_{\overline{1}}$, then $c_{1} x=\beta q_{1}+\gamma p_{1} \in B_{\overline{1}}$ and $(a+b)\left(c_{1} x\right)=(\gamma-\beta)\left(p_{1}-q_{1}\right) \subseteq B_{\overline{1}}$. Therefore if $\gamma \neq \beta$, then $\left\{p_{1}, q_{1}\right\} \subseteq B_{\overline{1}}$. So either $\left\{p_{1}, q_{1}\right\} \subseteq B_{\overline{1}}$ or $B_{\overline{1}} \subseteq F p_{1}+F q_{1}+F\left(p_{2}+q_{2}\right)$. Moreover with $\gamma \neq \beta, q_{1} x \notin B_{\overline{0}}$. Therefore $\gamma=\beta$ and $B=\left\langle e, f, a+b, c_{1}, p_{1}, q_{1}, p_{2}+q_{2}\right\rangle$, which is a maximal subalgebra.

(4) If $\operatorname{dim} V_{0}=3$, then $\operatorname{rank}\left(\left.Q\right|_{V_{0}}\right)=2$ or 3 .

If $\operatorname{rank}\left(\left.Q\right|_{V_{0}}\right)=3$, then we can suppose by Witt's Theorem and Theorem 3.3 that $V_{0}=F a+F b+F \cdot\left(c_{1}+c_{2}\right)$. Again $B_{\overline{1}}=P_{B}+Q_{B}$. If $\operatorname{dim} P_{B}=2$, then $\operatorname{dim} Q_{B}=2$ (because $\left.b P_{B} \subseteq Q_{B}\right)$ and $B_{\overline{1}}=\left(K_{10}\right)_{\overline{1}}$, a contradiction. So $\operatorname{dim} P_{B}=1=\operatorname{dim} Q_{B}$, but then, since $\left(c_{1}+c_{2}\right) B_{\overline{1}} \subseteq B_{\overline{1}}$ and $B_{\overline{1}} B_{\overline{1}} \subseteq B_{\overline{0}}$, it follows that either $B_{\overline{1}}=\left\langle p_{2}, q_{1}\right\rangle$ or $B_{\overline{1}}=\left\langle p_{1}, q_{2}\right\rangle$, and this contradicts $b B_{\overline{1}} \subseteq B_{\overline{1}}$.

If $\operatorname{rank}\left(\left.Q\right|_{V_{0}}\right)=2$, then $V_{0}=F a+F b+F c_{1}$ and $B_{\overline{1}}=P_{B}+Q_{B}$, as usual. If $\operatorname{dim} P_{B}=2$, then $\operatorname{dim} Q_{B}=2$, a contradiction. So $\operatorname{dim} P_{B}=1$ and $\operatorname{dim} Q_{B}=1$. Since $P_{B} Q_{B} \subseteq V_{0}$ we have that $B_{\overline{1}}=\left\langle p_{1}, q_{1}\right\rangle$ and $B=\left\langle e, f, a, b, c_{1}, p_{1}, q_{1}\right\rangle$, which is a maximal subalgebra of $K_{10}$.

Next we describe the structure of each one of the types of maximal subalgebras that we have obtained for $K_{10}$ :

(i) $\left(K_{10}\right)_{\overline{0}}$ is semisimple, and we have seen that $K_{10} \cong J(V, Q) \oplus F$.

(ii) $\left\langle e, f, a, p_{1}, p_{2}\right\rangle$ is semisimple and isomorphic to $F \oplus D_{-6}$, where $F \cong\langle 2 e-a\rangle$ and $\langle f, 2 e+a\rangle+\left\langle p_{1}, p_{2}\right\rangle \cong D_{-6}$.

(iii) $B=\left\langle e, f, a+b, c_{1}, p_{1}, q_{1}, p_{2}+q_{2}\right\rangle$ has the following radical:

$$
R=\left\langle c_{1}, a+b, p_{1}+q_{1}\right\rangle,
$$

and the quotient of the subalgebra $B$ by its radical is isomorphic to $D_{-3}$ :

$$
B / R=\left\langle\bar{e}, \bar{f}, \overline{p_{1}}, \frac{\overline{p_{2}+q_{2}}}{2}\right\rangle \cong D_{-3} .
$$


(iv) $B=\left\langle e, f, a, b, c_{1}, p_{1}, q_{1}\right\rangle$ has as radical $R=\left\langle c_{1}, p_{1}, q_{1}\right\rangle$ with $B / R \cong F \oplus T$ with $T$ the Jordan algebra of a bilinear form and

$$
B / R \cong\langle\bar{e}, \bar{f}, \bar{a}, \bar{b}\rangle .
$$

Finally, we will give the description of the maximal subalgebras of $K_{10}$ in terms of the realization shown in Section 2, given by Benkart and Elduque. First consider the map

$$
\begin{aligned}
& \varphi: K_{10} \quad \longrightarrow \quad K_{10} \\
& \lambda 1+a \otimes b \quad \lambda 1+(-1)^{\bar{a} \bar{b}} b \otimes a,
\end{aligned}
$$

with $a, b$ homogeneous elements of $K_{3}$. We remark that $\varphi$ is an automorphism because

$$
\begin{aligned}
\varphi(a \otimes b) \varphi(c \otimes d) & =\left((-1)^{\bar{a} \bar{b}} b \otimes a\right) \cdot\left((-1)^{\bar{c} \bar{d}} d \otimes c\right) \\
& =(-1)^{\bar{a} \bar{b}+\bar{c} \bar{d}+\bar{a} \bar{d}}\left(b d \otimes a c-\frac{3}{4}(b \mid d)(a \mid c) 1\right) \\
& =(-1)^{\bar{b} \bar{c}}\left((-1)^{(\bar{a}+\bar{c})(\bar{b}+\bar{d})}\left(b d \otimes a c-\frac{3}{4}(a \mid c)(b \mid d) 1\right)\right) \\
& =\varphi((a \otimes b)(c \otimes d)) .
\end{aligned}
$$

Corollary 4.2. The maximal subalgebras of $K_{10}=F \cdot 1 \oplus\left(K_{3} \otimes K_{3}\right)$ are, up to conjugation by automorphisms, the following:

(i) $\left(K_{10}\right) \overline{0}$.

(ii) The fixed subalgebra by the automorphism $\varphi$.

(iii) The subalgebra $F \cdot 1 \oplus\left(M \otimes K_{3}\right)$, where $M=F e+F x$ is a maximal subalgebra of $K_{3}$.

(iv) The subalgebra $F \cdot 1+F(e \otimes e)+\left(x \otimes K_{3}\right)+\left(K_{3} \otimes y\right)$.

Proof. It is easy to compute the fixed elements by $\varphi$ in terms of the original basis of $K_{10}$ given in the introduction. This subalgebra is $\left\langle e, f, c_{1}+c_{2}, p_{1}-q_{2}, p_{2}-q_{1}\right\rangle$, and now, using Proposition 3.1, we know that there exists an automorphism of $K_{10}$ that applies $\left\langle e, f, a, p_{1}, p_{2}\right\rangle$ into $\left\langle e, f, c_{1}+c_{2}, p_{1}-q_{2}, p_{2}-q_{1}\right\rangle$ (notice that $F\left(c_{1}+c_{2}\right)$ and $F a$ are isometric relative to the bilinear form $Q$ of $V$, and moreover $\left\langle p_{1}-q_{2}, p_{2}-q_{1}\right\rangle=$ $\left.\left\{x \in\left(K_{10}\right)_{\overline{0}} \mid\left(c_{1}+c_{2}\right) x=-x\right\}\right)$. So the maximal subalgebras of type (ii) in Theorem 4.1 are conjugate to the subalgebra of fixed elements by $\varphi$.

Also the maximal subalgebras of type (iii) correspond, through the isomorphism in Theorem 2.1, to $\langle 1, e \otimes e, x \otimes x, x \otimes y, e \otimes x, x \otimes e, e \otimes y\rangle$, that is, to subalgebras $F \cdot 1 \oplus\left(M \otimes K_{3}\right)$ with $M$ a maximal subalgebra of $K_{3}$, while the maximal subalgebras of type (iv) correspond, through the isomorphism in Theorem 2.1, to $F \cdot 1+F(e \otimes$ $e)+\left(x \otimes K_{3}\right)+\left(K_{3} \otimes y\right)$.

\section{REFERENCES}

[1] A. Albert, On a certain algebra of quantum mechanics, Ann. of Math. (2) 35 (1934), 65-73. MR.1503142

[2] G. Benkart, A. Elduque, A new construction of the Kac Jordan superalgebra, Proc. Amer. Math. Soc. 130 (2002) no.11, 3209-3217. MR1912998 (2003d:17024)

[3] E. Dynkin, Maximal subgroups of the classical groups, Trudy Moskov. Mat. Obsc., (1952), 39-166; Amer. Math. Soc. Transl. 6 (1957), 245-378. MR0049903 (14:244d)

[4] E. Dynkin, Semisimple subalgebras of semisimple Lie algebras, Mat. Sbornik 30 (1952), 249462; Amer. Math. Soc. Transl. 6 (1957), 111-244. MR0047629 (13:904c) 
[5] A. Elduque, On maximal subalgebras of central simple Malcev algebras, J. Algebra 103 (1) (1986), 216-227. MR860701 (88a:17043)

[6] A. Elduque, J. Laliena, S. Sacristán, Maximal subalgebras of associative superalgebras, J. Algebra 275 (2004), 40-58. MR2047439 (2005c:16064)

[7] A. Elduque, J. Laliena, S. Sacristán, Maximal subalgebras of Jordan superalgebras, arXiv:math.RA/0606017

[8] P. Jordan, J. von Neumann and E. Wigner, On an algebraic generalization of the quantum mechanical formalism, Ann. of Math. (2) 35 (1934), 29-64. MR1503141

[9] V.G. Kac, Classification of Simple Z-Graded Lie Superalgebras and Simple Jordan Superalgebras, Comm. Algebra 13 no.5 (1977), 1375-1400. MR0498755 (58:16806)

[10] I.L. Kantor, Connection between Poisson brackets and Jordan and Lie superalgebras, Lie Theory, Differential Equations and Representation Theory. Publications CRM, Montreal, 1990, 213-225. MR 1121965 (92f:17040)

[11] D. King, The split Kac Superalgebra $K_{10}$, Comm. Algebra 22 no.1 (1994), 29-40. MR.1255668 (95a:17014)

[12] K. McCrimmon, Speciality and Non-speciality of Two Jordan Superalgebras, J. Algebra 149 (1992), 326-351. MR1172433 (93k:17060)

[13] Yu.A. Medvedev, E.I. Zelmanov, Some counterexamples in the theory of Jordan algebras, in: Nonassociative Algebraic Models (Zaragoza 1989), S. González and H.C. Myung, eds.; Nova Sci. Publ., Commack, NY, 1992, 1-16.

[14] M. Racine, On Maximal subalgebras, J. Algebra 30 (1974), 155-180. MR0349771 (50:2264)

[15] M. Racine, Maximal subalgebras of exceptional Jordan algebras, J. Algebra 46 (1977), 12-21. MR0450356 (56:8651)

[16] M.L. Racine, E.I. Zelmanov, Simple Jordan Superalgebras with Semisimple Even Part, J. Algebra 270 (2003), 374-444. MR2019625 (2005b:17063)

[17] A.S. Shtern, Representation of an exceptional Jordan superalgebra, Funktsional Anal. i Prilozhen 21 (1987), 93-94 (Russian), English translation in Functional Anal. Appl. 21 (1987), 253-254. MR911787 (89b:17009)

[18] C.T.C. Wall, Graded Brauer Groups, Jour. Reine Angew. Math. 213 (1964), 187-199. MR0167498 (29:4771)

Departamento de Matemáticas, Universidad de Zaragoza, 50009 Zaragoza, Spain

E-mail address: elduque@unizar.es

Departamento de Matemáticas y Computación, Universidad de La Rioja, 26004, LOGROÑO, SPAIN

E-mail address: jesus.laliena@dmc.unirioja.es

Departamento de Matemáticas y Computación, Universidad de La Rioja, 26004, LOGROÑO, SPAIN

E-mail address: ssacrist@barpimo.com 